\title{
CAN UAV LIDAR DERIVE VERTICAL STRUCTURE OF HERBACEOUS VEGETATION ON RIVERDIKE?
}

\author{
Naoko Miura ${ }^{1}$, Tomoyo F. Koyanagi ${ }^{2}$, Shigehiro Yokota ${ }^{3}$, Susumu Yamada ${ }^{4}$ \\ ${ }^{1}$ Graduate School of Agricultural and Life Sciences, the University of Tokyo - miura@uf.a.u-tokyo.ac.jp \\ ${ }^{2}$ Field Studies Institute for Environmental Education, Tokyo Gakugei University - leaves23jp@gmail.com \\ ${ }^{3}$ Faculty of Environmental Studies, Tokyo City University - yokotas@tcu.ac.jp \\ ${ }^{4}$ Faculty of Agriculture, Tokyo University of Agriculture - sy206447@nodai.ac.jp
}

\section{KEY WORDS: UAV, LiDAR, Herbaceous vegetation, Grass, Riverdike}

\begin{abstract}
:
Herbaceous vegetation on riverdike is important to prevent soil erosion, which may lead to collapse of riverdikes and consequently severe flooding. To keep suitable vegetation condition and secure visibility of riverdike for inspection, managers need to know where thick and tall herbaceous vegetation grows on riverdikes in a cost effective manner. This paper aims to derive vertical structure of herbaceous vegetation on riverdike using UAV LiDAR. UAV LiDAR based indices, V-bottom, V-middle and V-top; presence of vegetation in bottom vegetation $(0-50 \mathrm{~cm})$, middle vegetation $(50-100 \mathrm{~cm})$ and top vegetation $(>100 \mathrm{~cm})$ respectively, were calculated and compared to field survey data for validation. The results showed that UAV LiDAR based assessment strongly correlated with field based assessment in middle and top vegetation with $\mathrm{R}^{2}=0.67$ and 0.85 , respectively. However, it displayed only moderate correlation in bottom vegetation $\left(\mathrm{R}^{2}=0.36\right)$. It was found that bottom vegetation might be underestimated when forb species or falling grass species is abundant in middle vegetation. However, underestimated place can be easily checked using proposed UAV LiDAR based V-bottom and V-middle values. The vertical structure information derived by proposed method would be valuable assets for vegetation management on riverdike.
\end{abstract}

\section{INTRODUCTION}

Riverdikes are built to protect human life and assets from flooding. They are huge, long river facilities and constantly embanked and repaired. The important function of herbaceous vegetation on riverdikes is to prevent soil erosion, which may lead to collapse of riverdikes and consequently severe flooding. Vegetation management is particularly crucial to keep suitable vegetation condition and secure visibility of riverdike for inspection, which is a labor-intensive work in itself. However, managers cannot conduct frequent mowing of vegetation any more due to recent reduction of management budget. This allows wide spread of invasive vegetation species, which makes visual inspection of riverdikes more difficult. Suitable vegetation management that improves the cost efficiency is required (Yamada and Nemoto, 2016). If managers can easily find where thick and tall herbaceous vegetation grows on riverdikes in a cost effective manner, it would greatly help vegetation management on riverdikes.

Light detection and ranging (LiDAR) is an active sensing technology that emits laser pulses and measures the range distance between sensor and the illuminated target. The technology provides 3D information of the target. The application of airborne LiDAR, which uses conventional manned fixed-wing aircraft or helicopter as a platform, for forestry and forest ecology has been well documented. Estimation of leaf area index (e.g. Zhao and Popescu, 2009), canopy cover (e.g. Coops et al., 2007), biomass (e.g. Næsset and Gobakken, 2008), stem number (e.g. Packalén and Maltamo, 2007) showed that LiDAR is a reliable tool for forest inventory. For extracting vertical structure of forest, Zimble et al. (2003) used LiDAR derived tree height variance to differentiate single-storey and multi-storey vertical structural classes with a $97 \%$ accuracy. Maltamo et al. (2005) tested the presence and the number of understorey trees by analysing the height distribution of LiDAR returns. Miura and Jones (2010) proposed a forest characterization scheme which estimates gaps and presence of vegetation in vertical layers by calculating LiDAR return types and their ratio. These studies again displayed the utility of LiDAR for forest ecology.

On the other hand, these technologies have rarely been applied to grassland. This is because LiDAR data collected using conventional aircraft does not assure accuracy and point density required for herbaceous vegetation analysis. Unmanned aerial vehicle (UAV) LiDAR is a new system which emerged lately in airborne laser scanning. The innovative feature of this system is to acquire data from relatively low flying altitude $(<100 \mathrm{~m})$ with more customized flight plan and low cost. It provides highly accurate and denser data than the conventional system. Wang et al. (2017) utilized UAV discrete LiDAR (Velodyne HDL-32E) to model canopy height and fractional cover in the cattle-grazing grassland, which were used to estimate above ground biomass in the same area. The authors indicated that the LiDAR derived indices are not very accurate for grassland ecosystem, but can be calibrated using the field data to predict the actual canopy height and fractional cover since there is relatively high level of correlation. In their study, average point density was not so high (26 points $/ \mathrm{m}^{2}$ ), which might be one of the explanations why the indices did not work very well. With more dense point cloud (460 points $/ \mathrm{m}^{2}$ ), Miura et al. (2018) produced a herbaceous vegetation height map on riverdike with spatial (vertical and horizontal) resolution of $5 \mathrm{~cm}$ using UAV waveform LiDAR (RIEGL VUX-1), and showed a potential of UAV LiDAR for grassland analysis. 
In this paper, we posed a question: can UAV LiDAR derive vertical structure of herbaceous vegetation on riverdike? Laser data acquired using UAV LiDAR is analysed to estimate vegetation density in vertical layers and compared with field survey data.

\section{METHODS}

\subsection{Study area}

The riverdike we studied locates in the high-water channel of the Tone River, $30 \mathrm{~km}$ northeast of Tokyo, Japan (Figure 1). Mean annual precipitation and temperature are $1344 \mathrm{~mm}$ and $14.1^{\circ} \mathrm{C}$, respectively at the nearby Ryugasaki Meteorological Station. Four sections of the riverdike are selected for the study and approximately $3 \mathrm{~km}$ long in total. The riverdike was approximately $7 \mathrm{~m}$ in height at the top and $40 \mathrm{~m}$ in width at the bottom with two sloped sides. Herbaceous vegetation covers both slopes of the riverdike. Tall herbaceous vegetation such as Imperata cylindrica (grass species) and Solidago altissima (forb species), which grow more than $1 \mathrm{~m}$ in height, are dominant species in this area. Mowing is carried out twice a year in midspring and late summer as a part of vegetation management by Tonegawa-Joryu River Office, Ministry of Land, Infrastructure, Transport and Tourism.

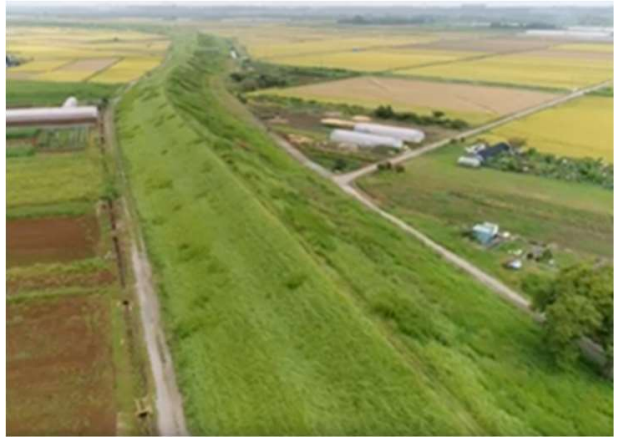

Figure 1. Riverdike in the high-water channel of the Tone River

\subsection{UAV Lidar data}

LiDAR data was acquired using UAV system of Nakanihon Air Service and Kohata Inc., TOKI which consists of GRYPHON DYNAMICS GD-X8-SP platform, RIEGL VUX-1 laser scanner and NIKON-TRIMBLE AP20 GNSS/IMU system (Figure 2). This is a rotary wing drone, quadrotor with a waveform laser system. The data was collected twice over the same area in August and October 2017 just before and after mowing. The duration of one flight was 12 minutes. Flights were repeated to cover whole study section of the riverdike. The flight altitude was $30 \mathrm{~m}$. This was chosen relative to the height of the riverdike and the desired point density. Table 1 shows the specifications for the laser data acquisition. Ground control points were surveyed using network RTK-GNSS for validation in height. Vertical accuracy of the acquisition was RMSE $2.1 \mathrm{~cm}$ (August) and $3.4 \mathrm{~cm}$ (October).

\subsection{Vegetation survey}

Vegetation survey was conducted in accordance with LiDAR data acquisition in August 2017. A circular plot of $50 \mathrm{~cm}$ radius was set up on the slope of the riverdike for vegetation survey. Survey marker was set up at the center of the plot to be recognized in the LiDAR data later. Twenty-five sites were selected based on vegetation composition. For each plot, vegetation cover was visually assessed as a percentage in three vertical categories; $0-50 \mathrm{~cm}, 50-100 \mathrm{~cm}$ and $100-\mathrm{cm}$ respectively. The dominant species were also recorded.

\begin{tabular}{lc}
\hline Sensor & RIEGL VUX-1 \\
Pulse repetition frequency & $50 \mathrm{kHz}$ \\
Scan angle & $330^{\circ}$ \\
Platform altitude & $30 \mathrm{~m}$ \\
Flying speed & $15 \mathrm{~km} / \mathrm{h}$ \\
Overlap between courses & $50-60 \%$ \\
Average point density & 460 points $/ \mathrm{m}^{2}$ \\
Acquisition date & August and October 2017 \\
\hline
\end{tabular}

Table 1. Specifications for the LiDAR data acquisition

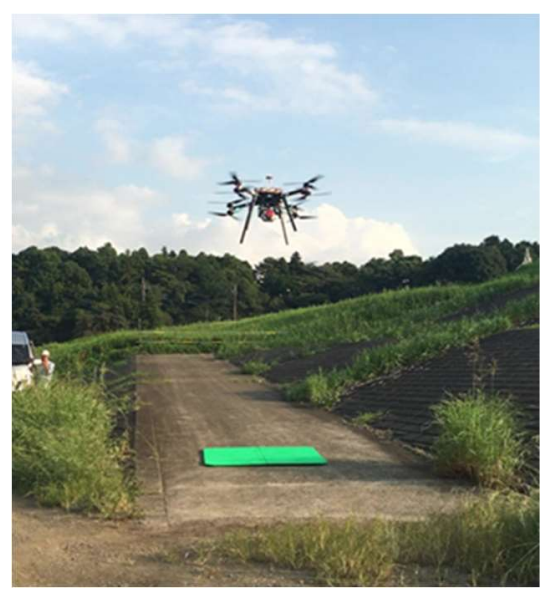

Figure 2. UAV LiDAR, TOKI

\subsection{Vertical structure of herbaceous vegetation}

In order to evaluate vertical structure of herbaceous vegetation on riverdike, we modified the forest characterization scheme proposed by Miura and Jones (2010). The authors vertically stratified forests into four layers; Ground, low vegetation (0-1 m from the ground), medium vegetation (1-5 $\mathrm{m}$ from the ground) and high vegetation $(>5 \mathrm{~m})$, and calculated LiDAR return ratio using pulse types (Type $1=$ singular returns, Type $2=$ first of many returns, Type $3=$ intermediate returns and Type $4=$ last of many returns) to evaluate forest structure (Table 2). OG, OL and $\mathrm{OM}$ represent gaps, that is, opening above Ground, low vegetation and medium vegetation layers respectively and VL, $\mathrm{VM}$ and VH show the presence of vegetation in low, medium and high vegetation layers in the forest respectively. CC exhibits horizontal coverage of canopy and DH displays how vertically dense the canopy is. Since we aim to evaluate herbaceous vegetation on riverdike instead of forests where more complex structure is composed by trees, shrubs and understory vegetation, we calculated only three categories; V-bottom (presence of bottom vegetation) modified from VL, V-middle (presence of middle vegetation) modified from VM and V-top (presence of top vegetation) modified from VH (Table 3 ). We chose a $50 \mathrm{~cm}$ and $100 \mathrm{~cm}$ threshold for herbaceous vegetation and classified LiDAR data into four layers; Ground, bottom vegetation (0-50 $\mathrm{cm})$, middle vegetation $(50-100 \mathrm{~cm})$ and top vegetation $(>100$ $\mathrm{cm}$ ) (Figure 3). In this process, Ground data was defined as all returns collected after mowing, and all returns acquired before mowing were classified into V-bottom, V-middle and V-top 
according to the height from the ground. Then, LiDAR return ratio was calculated. The total number of returns, $T$ is expressed:

$$
T=\sum_{i=1}^{i=4} \sum_{J=1}^{4} R_{i j}
$$

where

$$
R=\mathrm{UAV} \text { LiDAR returns }
$$

$i=$ classified 4 layers $(1=$ top vegetation, $2=$ middle vegetation, $3=$ bottom vegetation and $4=$ Ground)

$j=$ return types $(1=$ Type $1,2=$ Type $2,3=$ Type 3 and $4=$ Type 4$)$

V-bottom comprises all return types (Type 1, 2, 3 and 4) from bottom vegetation layer; this represents the presence of bottom vegetation:

$$
\text { V-bottom }=\frac{R_{31}+R_{32}+R_{33}+R_{34}}{T}=\frac{\sum_{J=1}^{4} R_{3 j}}{T}
$$

V-middle contains all return types (Type 1, 2, 3 and 4) from middle vegetation layer; this indicates the presence of middle vegetation:

$$
\text { V-middle }=\frac{R_{21}+R_{22}+R_{23}+R_{24}}{T}=\frac{\sum_{J=1}^{4} R_{2 j}}{T}
$$

V-top comprises all return types (Type 1, 2, 3 and 4) from top vegetation layer; this represents the presence of top vegetation:

$$
\text { V-top }=\frac{R_{11}+R_{12}+R_{13}+R_{14}}{T}=\frac{\sum_{J=1}^{4} R_{1 j}}{T}
$$

\begin{tabular}{|c|c|c|c|}
\hline Category & & Description & LiDAR return ratio \\
\hline 1 & OG & $\begin{array}{l}\text { Opening above the } \\
\text { ground }\end{array}$ & Ground Type 1 \\
\hline 2 & $\mathrm{OL}$ & $\begin{array}{l}\text { Opening above low } \\
\text { vegetation }\end{array}$ & $\begin{array}{l}\text { Type } 1 \& 2 \text { in low } \\
\text { vegetation }\end{array}$ \\
\hline 3 & VL & $\begin{array}{l}\text { Presence } \\
\text { understorey } \\
\text { vegetation }\end{array}$ & $\begin{array}{l}\text { Total (Type } 1,2,3,4) \text { in } \\
\text { low vegetation }\end{array}$ \\
\hline 4 & $\mathrm{CC}$ & Canopy cover & $\begin{array}{l}\text { Type } 1 \& 2 \text { in medium } \\
\text { and high vegetation }\end{array}$ \\
\hline 5 & $\mathrm{OM}$ & $\begin{array}{l}\text { Opening above } \\
\text { medium vegetation }\end{array}$ & $\begin{array}{l}\text { Type } 1 \& 2 \text { in medium } \\
\text { vegetation }\end{array}$ \\
\hline 6 & VM & $\begin{array}{l}\text { Presence of med- } \\
\text { storey vegetation }\end{array}$ & $\begin{array}{l}\text { Total (Type } 1,2,3,4 \text { ) in } \\
\text { medium vegetation }\end{array}$ \\
\hline 7 & $\mathrm{VH}$ & $\begin{array}{l}\text { Presence of high } \\
\text { trees }\end{array}$ & $\begin{array}{l}\text { Total (Type } 1,2,3,4) \text { in } \\
\text { high vegetation }\end{array}$ \\
\hline 8 & $\mathrm{DH}$ & $\begin{array}{l}\text { Vertically dense } \\
\text { canopy of high trees }\end{array}$ & $\begin{array}{l}\text { Type } 3 \& 4 \text { in high } \\
\text { vegetation }\end{array}$ \\
\hline
\end{tabular}

These ratios are expressed in percentage and subsequently compared to the vegetation survey data to assess its utility in characterizing vertical structure of herbaceous vegetation.

Table 2. Forest Characterization Scheme (after Miura and Jones, 2010)

\begin{tabular}{llll}
\hline Category & & Description & LiDAR return ratio \\
\hline 1 & $\begin{array}{l}\text { V- } \\
\text { bottom }\end{array}$ & $\begin{array}{l}\text { Presence of less } \\
\text { than } 50 \mathrm{~cm} \text { height } \\
\text { vegetation }\end{array}$ & $\begin{array}{l}\text { Total (Type 1,2,4) in } \\
\text { bottom vegetation }\end{array}$ \\
2 & $\begin{array}{l}\text { V- } \\
\text { middle }\end{array}$ & $\begin{array}{l}\text { Presence of } 50- \\
100 \mathrm{~cm} \text { height } \\
\text { vegetation } \\
\text { Presence of more } \\
\text { than } 100 \mathrm{~cm} \text { height } \\
\text { vegetation }\end{array}$ & $\begin{array}{l}\text { Total }(\text { Type } 1,2,4) \text { in } \\
\text { middle vegetation }\end{array}$ \\
top vegetation $1,2,4)$ in \\
\hline
\end{tabular}

Table 3. Modified characterization scheme to derive vertical structure of herbaceous vegetation

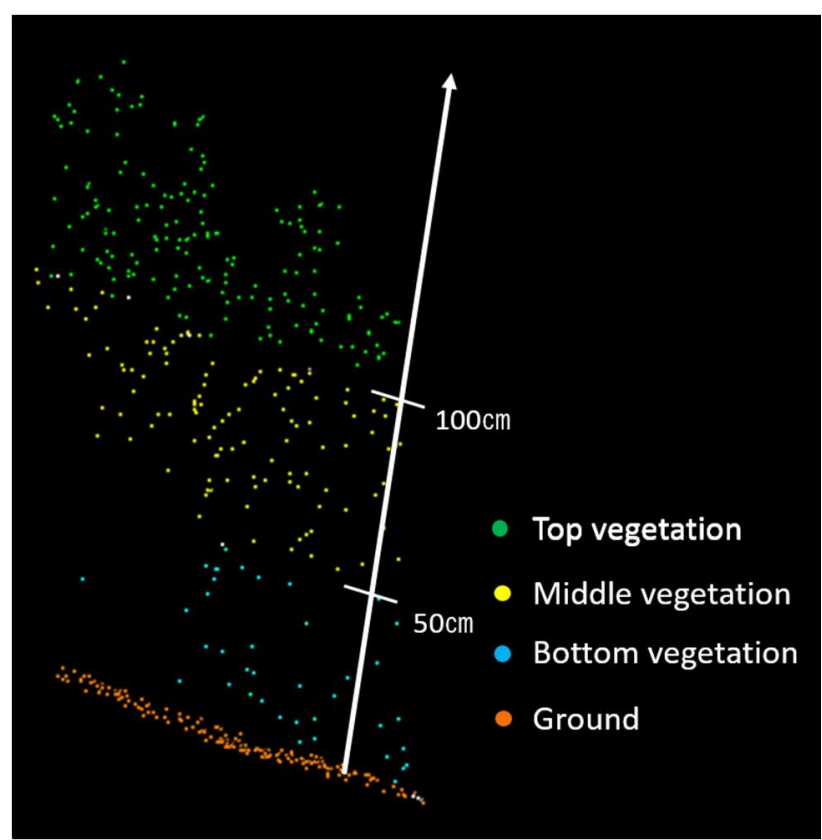

Figure 3. LiDAR point cloud classification. LiDAR point cloud was classified into four layers; Ground, which contains all returns collected after mowing, bottom vegetation $(0-50 \mathrm{~cm})$, middle vegetation $(50-100 \mathrm{~cm})$ and top vegetation $(>100 \mathrm{~cm})$, which were derived from the data collected before mowing

\section{RESULTS}

\subsection{Point cloud in vegetation plot}

Figure 4 presents the number of returns in Ground, bottom vegetation $(0-50 \mathrm{~cm})$, middle vegetation $(50-100 \mathrm{~cm})$ and top vegetation $(>100 \mathrm{~cm})$ layers in each vegetation plot. The total number of returns was large (> 800) in Plot 1, 2, 3, 4, 5, 6 and 16 where grass species, Imperata cylindrica was mostly dominant. Bottom and middle vegetation were found in every plot, however, top vegetation was found mostly in the plot where forb species, Solidago altissima was dominant. As a result of type classification, it was found that approximately $99.1 \%$ was Type 1 (singular returns). Type 2 (first of many returns) and Type 4 (last of many returns) were approximately $0.4 \%$ and $0.5 \%$ respectively. There was no Type 3 in 25 vegetation plots.

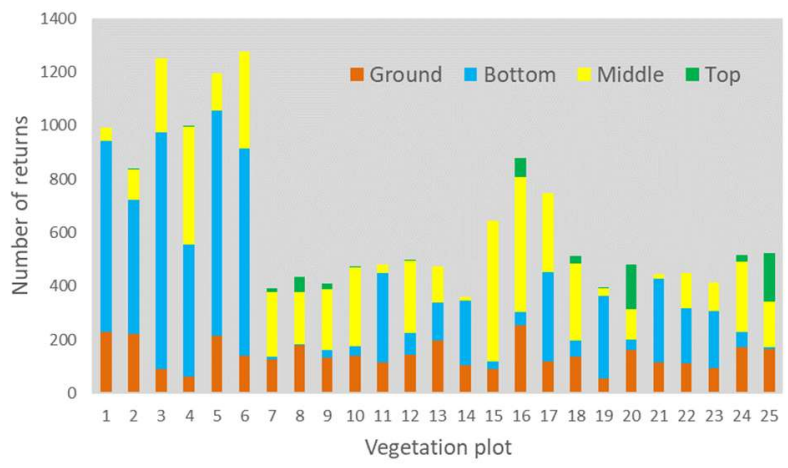

Figure 4. Number of returns in Ground, bottom vegetation (0-50 $\mathrm{cm})$, middle vegetation $(50-100 \mathrm{~cm})$ and top vegetation $(>100$ $\mathrm{cm})$ layers in each vegetation plot 
3.2 Comparison of vertical vegetation structure between UAV LiDAR based and field based assessment

UAV LiDAR based V-bottom showed a moderate correlation with field based vegetation cover less than $50 \mathrm{~cm}$ in height with $\mathrm{R}^{2}=0.36$ (Figure 5a). UAV LiDAR based V-middle displayed a good correlation with field based vegetation cover in middle vegetation $(50-100 \mathrm{~cm})$ with $\mathrm{R}^{2}=0.67$ (Figure $5 \mathrm{~b}$ ). These associations were observed across a range of vegetation cover. UAV LiDAR based V-top and field based vegetation cover more than $100 \mathrm{~cm}$ in height was significantly correlated with $\mathrm{R}^{2}=0.85$ (Figure 5c).
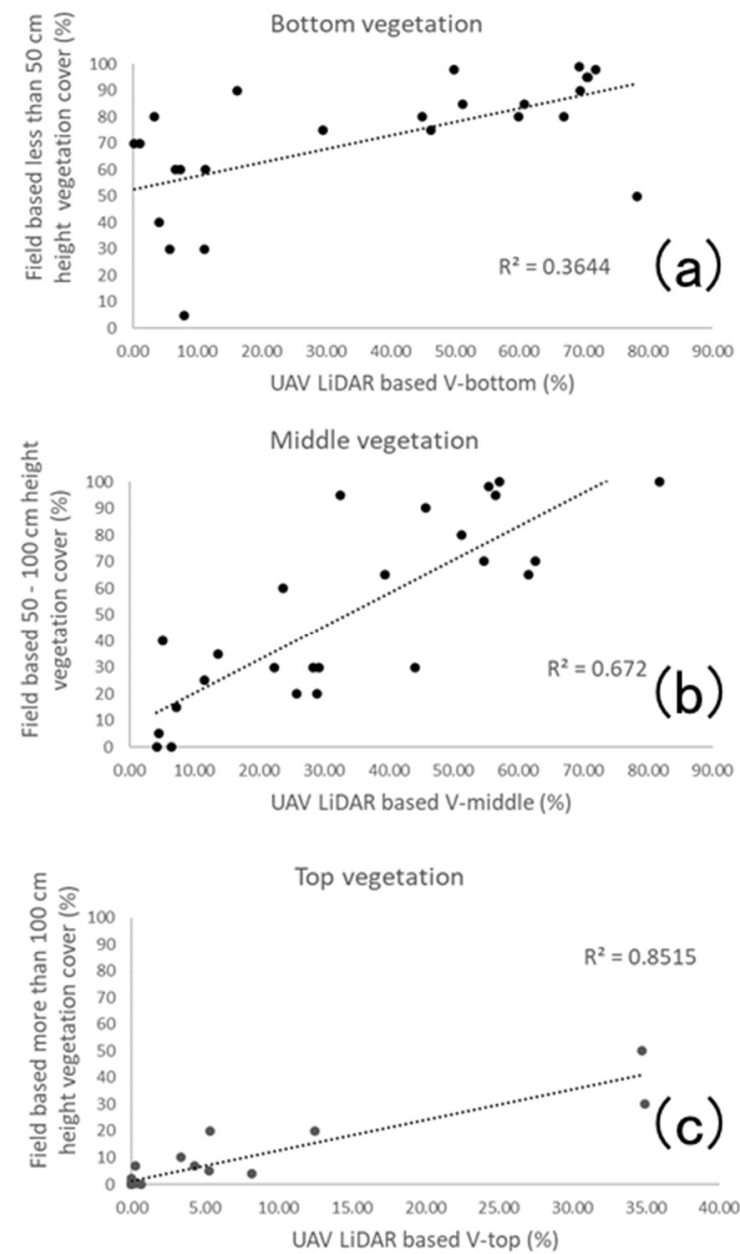

Figure 5. Comparison between UAV LiDAR based (a)V-bottom, (b)V-middle, (c)V-top and field based vegetation cover

\section{DISCUSSION}

The analysis of point cloud in the vegetation plots revealed that UAV LiDAR can acquire sufficient returns for analysis of vertical structure of herbaceous vegetation. It was found that most returns are singular returns, although the laser sensor has ability to record multiple returns. Most of herbaceous vegetation in the vegetation plots was less than $100 \mathrm{~cm}$ in height. For such micro environment, detecting multiple returns might be difficult even for UAV waveform LiDAR.

UAV LiDAR based assessment worked well to derive vertical structure of herbaceous vegetation in middle $(50-100 \mathrm{~cm})$ and top vegetation $(>100 \mathrm{~cm})$. This would be valuable information for vegetation management on riverdike. Managers can efficiently find where tall and dense vegetation grows on enormous riverdike, where maintenance would be most likely necessary. On the other hand, UAV LiDAR based V-bottom did not show good correlation with field based vegetation cover in bottom vegetation $(0-50 \mathrm{~cm})$. Profiles of UAV LiDAR returns in the vegetation plots suggest that some plots did not record returns properly in bottom vegetation layer while others did (Figure 6). In herbaceous vegetation environment, when some vegetation is present in middle vegetation layer, there should be similar amount or more vegetation present in below layer; bottom vegetation. However, some plots showed too few UAV LiDAR returns in bottom vegetation layer when compared to the number of returns in middle vegetation layer. This anomaly can be detected when compared UAV LiDAR based V-bottom with Vmiddle (Figure 7). The plots, which show too few V-bottom values compared to their $\mathrm{V}$-middle values, should have a problem to assess vegetation structure in bottom vegetation. With reference photo and vegetation survey data, we confirmed that those 11 plots with anomaly were dominated by forb species such as Solidago altissima (Figure 6c), Cayratia japonica (Figure 8a), Artemisia indica var. maximowiczii (Figure 8b) and Bidens pilosa

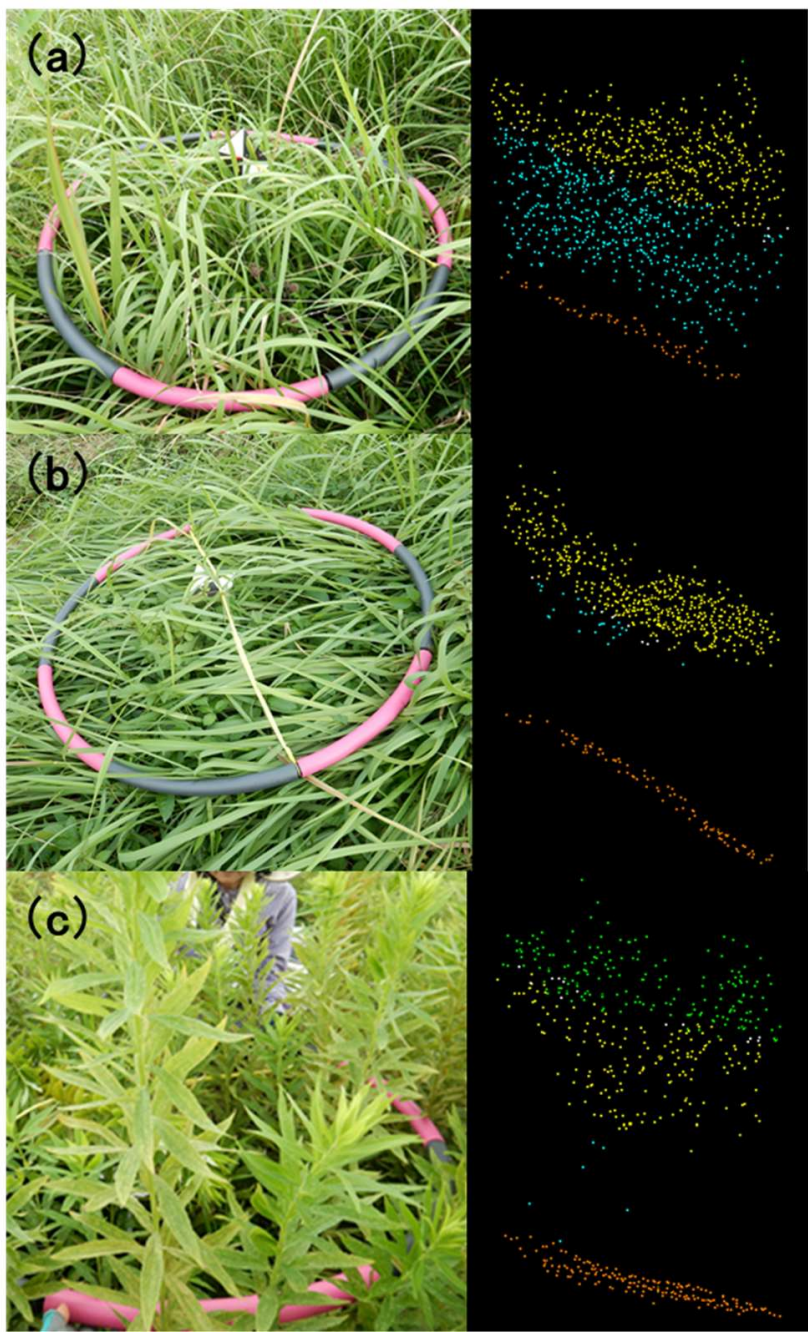

Figure 6. Reference photo (left) and profile of UAV LiDAR returns (right) in vegetation plots. UAV LiDAR returns in bottom vegetation layer (blue points) were properly recorded in (a) Imperata cylindrica (grass species) dominated plot, however, they were scarce in (b) falling Imperata cylindrica (grass species) and (c) Solidago altissima (forb species) dominated plots. 


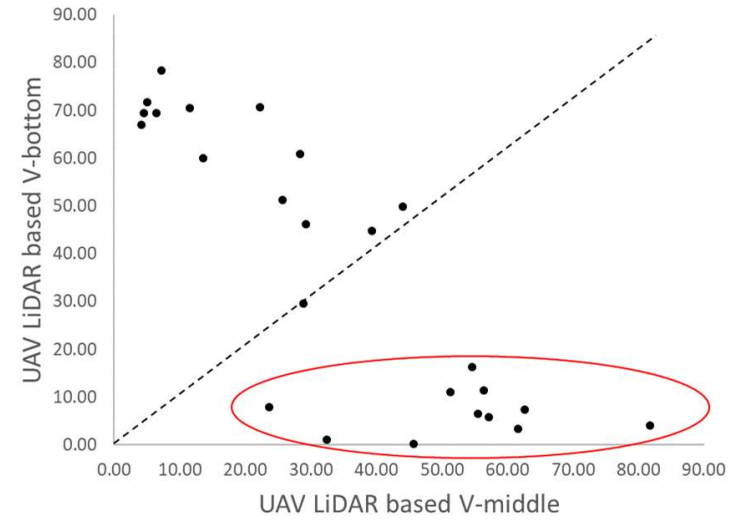

Figure 7. Comparison between UAV LiDAR based V-bottom and V-middle in 25 vegetation plots. Circled plots show too few $\mathrm{V}$-bottom values compared to their V-middle values.

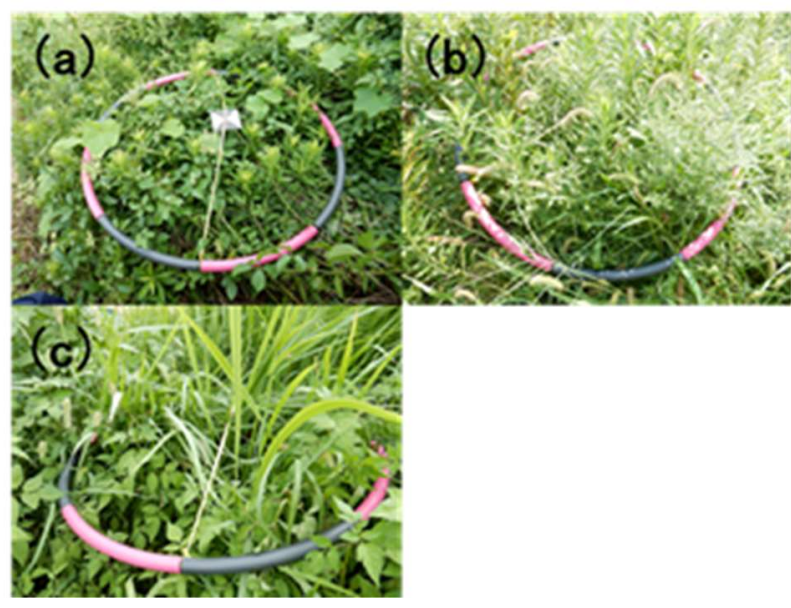

Figure 8. Forb species which prevent UAV LiDAR penetration into bottom vegetation layer $(0-50 \mathrm{~cm})$ when they are present in middle vegetation layer $(50-100 \mathrm{~cm})$.

var. pilosa (Figure 8c). As an exception, two plots were dominated by grass species, but "falling" Imperata cylindrical (Figure 6b). These species were thick in middle vegetation layer. This suggests that broad leaves of forb species or sides of leaves of falling grass species physically prevent UAV LiDAR penetration into bottom vegetation layer when they are abundantly present in middle vegetation layer. This assumption fits with the result of total number of returns in each vegetation plot (Figure 4). The plots where "upright" grass species are dominant tend to have much more returns than plots with forb species or "falling" grass species. Morphology of herbaceous vegetation in middle vegetation layer would be a key to derive complete vertical structure of herbaceous vegetation.

The relationship between field based and LiDAR based vegetation assessment was well correlated (Figure 5), which suggests that LiDAR based assessment can be regressed by field data. We used visually assessed vegetation cover as the field data for efficiency in the field survey, however, this might not be the best to regress with LiDAR based assessment. Values of field based vegetation cover displayed a tendency to be higher than that of LiDAR based assessment. As is often the case with visual assessment in the field, values of vegetation cover might have been overestimated in the field. To eliminate uncertainty in the vegetation survey, more systematic and quantitative survey methods are necessary. Since LiDAR based assessment represents the presence of vegetation in vertical layers, biomass values derived from the methods such as stratified clipping methods, which are more systematic but require much more time and labour in the field, might be more suitable for regression analysis with LiDAR based assessment. Further investigation is required for this supposition.

\section{CONCLUSION}

In conclusion, UAV LiDAR is a promising tool to derive vertical structure of herbaceous vegetation. Proposed method successfully represents middle vegetation $(50-100 \mathrm{~cm})$ and top vegetation $(>100 \mathrm{~cm})$. This would be an important information for vegetation management on riverdike. It was found that bottom vegetation $(0-50 \mathrm{~cm})$ might be underestimated when forb species or falling grass species is abundant in middle vegetation. However, underestimated place can be easily checked using proposed UAV LiDAR based V-bottom and V-middle values.

\section{ACKNOWLEDGEMENTS}

This research was supported by JSPS KAKENHI Grant Number 17H03958. We thank Tonegawa-Joryu River Office, Ministry of Land, Infrastructure, Transport and Tourism for their support. We also acknowledge Yoshiko Kitagawa for her assistance in vegetation survey.

\section{REFERENCES}

Coops, N., Hilker, T., Wulder, M., St-Onge, B., Newnham, G., Siggins, A., Trofymow, J., 2007. Estimating canopy structure of Douglas-fir forest stands from discrete-return LiDAR. Trees Structure and Function 21, pp. 295-310.

Maltamo, M., Packalen, P., Yu, X., Eerikainen, K., Hyyppa, J., Pitkanen, J., 2005. Identifying and quantifying structural characteristics of heterogeneous boreal forests using laser scanner data. Forest Ecology and Management 216, pp. 41-50.

Miura, N., Jones, S.D., 2010. Characterizing forest ecological structure using pulse types and heights of airborne laser scanning. Remote Sensing of Environment 114, pp. 1069-1076.

Miura, N., Yokota, S., Koyanagi, T.F., Yamada, S., 2018. Herbaceous vegetation height map on riverdike derived from UAV LiDAR data, 2018 IEEE International Geoscience and Remote Sensing Symposium (IGARSS).

Næsset, E., Gobakken, T., 2008. Estimation of above- and below-ground biomass across regions of the boreal forest zone using airborne laser. Remote Sensing of Environment 112, pp. 3079-3090.

Packalén, P., Maltamo, M., 2007. The k-MSN method for the prediction of species-specific stand attributes using airborne laser scanning and aerial photographs. Remote Sensing of Environment 109, pp. 328-341.

Wang, D., Xin, X., Shao, Q., Brolly, M., Zhu, Z., Chen, J., 2017. Modeling Aboveground Biomass in Hulunber Grassland Ecosystem by Using Unmanned Aerial Vehicle Discrete Lidar. Sensors 17, p. 180.

Yamada, S., Nemoto, M., 2016. Effects of Bare-Ground Revegetation Techniques Using Imperata cylindrica on Changes in the Plant Cover and Species Richness during Early Succession. Open Journal of Ecology Vol.06, pp. 471-483. 
Zhao, K., Popescu, S., 2009. Lidar-based mapping of leaf area index and its use for validating GLOBCARBON satellite LAI product in a temperate forest of the southern USA. Remote Sensing of Environment 113, pp. 1628-1645.

Zimble, D.A., Evans, D.L., Carlson, G.C., Parker, R.C., Grado, S.C., Gerard, P.D., 2003. Characterizing vertical forest structure using small-footprint airborne LiDAR. Remote Sensing of Environment 87, pp. 171-182. 